\title{
Fingerprint Pattern Recoqnition Using LVQ
}

\author{
Muhammad Donni Lesmana Siahaan ${ }^{1}$, Andysah Putera Utama Siahaan ${ }^{2}$ \\ Faculty of Computer ScienceUniversitas Pembangunan Panca Budi \\ Jl. Jend. Gatot Subroto Km. 4,5 Sei Sikambing, 20122, Medan, Sumatera Utara, Indonesia
}

\begin{abstract}
Various ways are developed to determine a person's personality. One was the use of neural networks to determine the human fingerprint patterns. This method is associated with dermatoglyphics. Dermatoglyphics is the study of human fingerprints and relation to aspects of a person's behavior. Fingerprint pattern everyone has its uniqueness. According to Dermatoglyphics science, fingerprint patterns can be used to understand the nature of a person's inborn. These properties include self-potential, personality, and preferences of the person.Meanwhile, the thickness and size of a person's fingerprint will continue to increase with age. However, will not change the pattern and shape of the fingerprint itself, unless the person has an accident that causes severely injured the finger.Learning Vector Quantization can be used to recognize the shape of a person's fingerprint.It helps the dermatoglyphics to determine the model of the personality of the person.
\end{abstract}

Keywords: LVQ, Dermatoglyphics, Fingerprint, Neural Network

\section{Introduction}

Developments in the field of information technology is very fast, especially in the field of the digital world [1][2]. The changes have had a considerable impact. One of them with their digitalized image data. With the image processing, a variety of patterns can be recognized easily. One of them is the shape of the fingerprint pattern. Fingerprints have a pattern of its own, especially in the ridges of the fingerprint. Differences in the pattern of the fingerprint can be used as identification of humans. With the fingerprint, the data themselves may not be confused with other data.

Biometric identification is always applied to check the authenticity of a person to enter into a security system. The identification of fingerprint pattern can be applied by using fuzzy techniques [7][8][9][10] as well. Fingerprints are one of the patterns used for biometric scanning. Fingerprints are used because they are the same, none of the people on this earth. Fingerprint pattern is unique. It is also often used for someone to recognize the nature of the human being. There are several categories of fingerprint patterns that reflect specific properties. To test these properties need a system that can recognize a person's fingerprint pattern. The introduction of the system requires trials and samples to reduce errors. Pattern recognition algorithms that involve certain developing very rapidly. This development created the science of artificial neural networks in pattern recognition fingerprint. It can perform fingerprint pattern recognition process with two stages, learning, and testing. This model is the model of how the human thinks. The algorithm used is Learning Vector Quantization (LVQ) which is an artificial neural network supervised learning process. This algorithm is expected fingerprint recognition can be accomplished quickly and accurately.In this study, LVQ will be used to train the fingerprint data by a certain amount and then will be compared with fingerprints tested. The experiments are performed by comparing the variable value changes with the degree of fit LVQ fingerprint patterns from the sample.

\section{Theories}

Fingerprints have four basic dermatoglyphic patterns already formulated. The pattern is a Whorl or Swirl, Arch, Loop, and Delta. Besides, some other patterns are variations of the four combinations of these patterns.

Whorl

Whorl is a form of spiral, bulls-eye, or double loopfingerprint as shown in Figure 1. Whorl has dots stand out and contrast and can be seen easily. The spiral pattern and a bulls-eye are exactly congruent in its interpretation, but that both give a little more focus. Everywhere in the hands, whorl highlight and emphasize to specific regions, making it a priority area in living subjects.

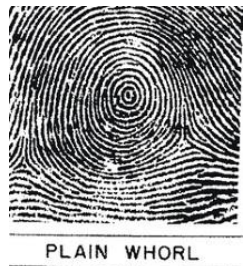

Fig. 1 Whorl Fingerprint 
Arch

The Arch pattern as shown in Figure 2 is seen as a Flat Arch is or Tented Arch. The arch pattern indicates traditional values and high morals. In almost all cases, the moral values are, to some people in the past in which they have been humiliated. People with this pattern having trouble seeing the negative traits of their own, and to understand that the past that they lock on the stain or the shame is just an experience that is required for the full development of personality. People with flat Arch follow the tradition with a bit of independent thinking, while those with patterns Tented Arch revealed an intellectual depth.

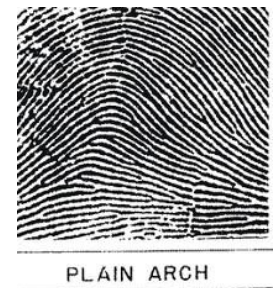

Fig. 2 Arch Fingerprint

Loop

It can rise toward the fingertips, or down towards the wrist. Common Loop move toward the thumb, while Radial Loop moves directing the tip to the side of the finger. The most common type of fingerprint is Common Loop. These patterns reveal the ability to use a variety of ideas from various sources of ideas, and combine these ideas with their way. Loop expresses a natural follower. The desire to lead others more often. However, not everyone has a common pattern Loop can lead.

\section{Radial Loop}

Radial Loop is the pattern swooping entering and departing from the thumb side of the hand. If the Common Loop show a mix of other styles, Radial Loop reveals the ability to create a style or an entirely new system. This person has a visual memory sharp, able to remember not only images but also actions and emotions that accompany the images earlier. As with all other signs, Radial Loop applies to any field or finger on it was found that sign.

\section{Double Loop}

Double Loop mostly misunderstood by almost all markings dermatoglyphic. In general, interpret the same as the Double Loop-whorl whorl another, with the main difference: Up to personality developed will tend strongly towards the statement exaggeration, manipulation, and subversive actions in the area of life. For example, someone with Double Loop on her thumbs may be in his early use of deception to help fulfill their wishes against the other. The owner of this hand lines drawn toward an exciting career, which with ordinary effort can be realized easily.

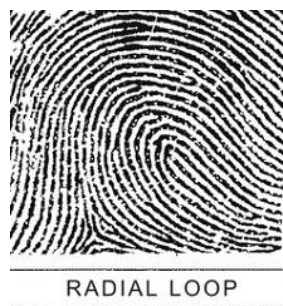

Fig. 3 Radial Loop Fingerprint

Delta

Delta is a cluster that contained in the estuary of the river water flowing into the sea or lake is always carrying mud and stones that eventually formed a group of islands called delta. Delta actual fingerprint is a point / line found on the center line of type parting lines. Delta is a focal point located at the front center of the separation of the staple lines. Staple line painting is the two deepest lines of some lines are lined up (parallel) and separating and (likely) covers basic painting (pattern area). Principal painting is a regional / white room surrounded by a line of type lines in which the room is a painting of lines fingerprints. In reality, not all fingerprints have Delta, but there is also a fingerprint that has more than one delta. Figure 4 show where the delta is. 


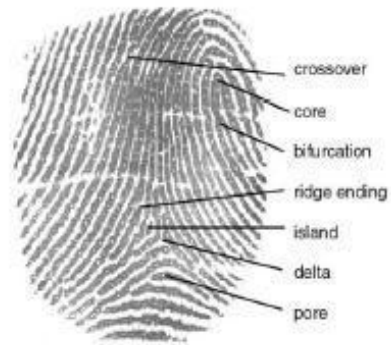

Fig. 4 Delta Fingerprint

\section{Related Works}

Previous research has been done that has been linked to personality analysis or fingerprint analysis. Shitala Prasad [4] predict a person's personality by analyzing handwriting in a digital form using the six traits that the font size, the slope of the letters and words, basic words, writing pressure, the space between letters and words. Experiments carried out in two stages, the first stage (A) using $2 / 3$ of the sample data as training data and $1 / 3$ the data as test data. This stage uses the 100 respondents, of which two-thirds of the sample data and the results of psychological handwriting used to train the system and the data $1 / 3$ handwriting samples tested to determine its accuracy, the results obtained $90.3 \%$ accuracy. The second phase (B) using the data as a test data and sample data used to train the rest of the system, the accuracy of the results obtained $93.86 \%$. The linkages quantitative parameters Dermatoglyphics, i.e., the number of strokes lines (ridge) a-b fingerprints, and ATD angle to diagnose patients with bronchial asthma [5]. Some ridges a-b high, can be used as diagnostic criteria for patients with bronchial asthma.

Thefingerprint patterns is to identify the level of intelligence with parameters ridge count (the number of strokes fingerprints), the number of strokes (ridges) between triradius A, B, C, D on the palms and the left hand corner of the ATD with the right hand. The sample used were70 people who had a high IQ (superior intelligence) and the 400 people who have moderate or ordinary intelligence. The method used is the method of statistical analysis of variance and discriminant analysis conclusions obtained is a level of intelligence to grow in line with increasing ridge count (strokes fingerprints) and a decrease in the angle ATD palms [3].The fingerprint classification method designed agglomerative hierarchical clustering techniques with FPROCK method that classifies the image with the concept of neighborhood each object [6]. The performance of the classification technique is measured by False Acceptance Rate and accuracy dataset variety of sizes. FPROCK technique can identify 6 class at $97.3 \%$ accuracy rate. While other classification techniques can be classified only five classes with an accuracy of $80 \%$ to $94.8 \%$.

\section{Methodology}

Learning Vector quantization is a method in the Neural Network to conduct supervised learning on the layer. Pattern classification methods with each unit-output represent a particular class or a particular category. Weight vector of an output unit that became the benchmark for the class / category is represented by the output. The approach taken is to classify the input vector based on the proximity of the input vector to the weights.

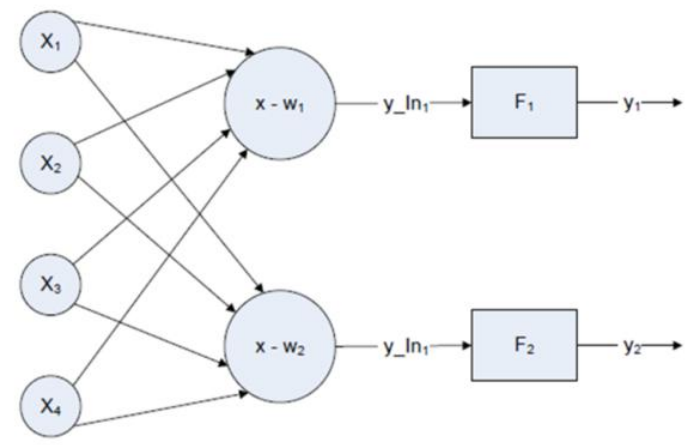

Fig. 5 LVQ Architecture

Figure 5 is an example of LVQ network structure which has four input layer with two units of neurons in the output layer. W1 and W2 are the weight that connects the input layer to the output layer. Each activation function F mapping each $y_{-} I n$ to classification $y 1$ or $y 2$. In F1, if $|x-w 1|<|x-w 2|$ then $y_{-}$In1 mapped to $y 1=1$ and mapped to $\mathrm{y} 1=0$ if otherwise. This condition also applies in F2, with appropriate conditions. 
LVQ Algorithm:

* Initialization

- Initialization of Vector Reference

- Learning rate initialization alpha $=0$

* If the condition of the wrong stop, do steps 2-6

* For each training vector, do steps 3-4

* Get the value j that $\|X\|$ wj minimum worth

* Update the weights wj

○ If $\mathrm{T}=\mathrm{Cj}$ then $w \mathrm{wj}=w \mathrm{wj}+\alpha(\mathrm{x}-\mathrm{wj})$

$\circ \quad$ If $\mathrm{T} \neq \mathrm{Cj}$ then $w \mathrm{j}=\mathrm{wj}-\alpha(\mathrm{x}-\mathrm{wj})$

* Update the value of learning rate

* Test the stop condition

Test the stop condition can be made based on a certain number of iterations (from step 1) or after the pace of learning has reached a very small price.

Where:

$\mathrm{X}=$ training vector

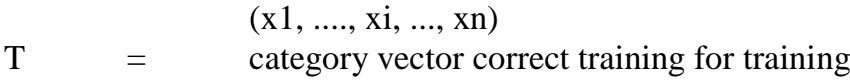

$\mathrm{Wj} \quad=\quad$ weight vector for the $\mathrm{j}$-th output unit

(w1j,...,wij, ....., win)

$\mathrm{Cj} \quad=\quad$ category or class represented by the unit value

of the output $\mathrm{j}$

$\| \mathrm{X}$-wj $\|=\quad$ Euclidean distances between vectors and vector enter the weight of the unit output $j$.

\section{Evaluation}

In this section, there are three fingerprint samples. There are Arch, Loop and Whorl. The dimension is $128 \mathrm{x} 128$ pixel. These figures below represent the samples.

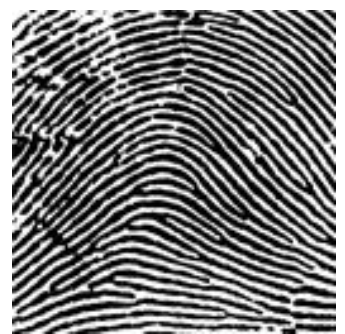

Fig. 6 Arch

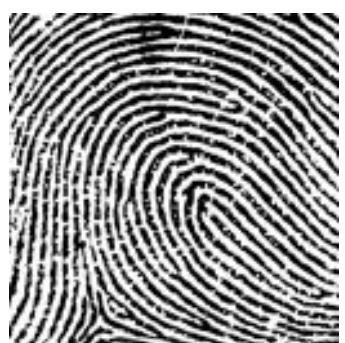

Fig. 7 Loop

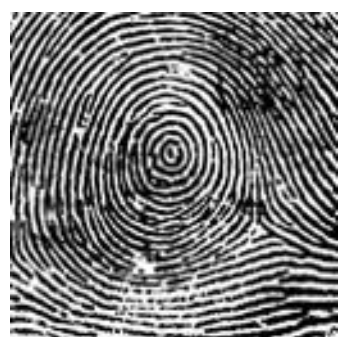

Fig. 8 Whorl 
The samples are converted into binary vector (v[0], v[1],..,v[16383]). There are 16384 numbers to be filled with 0 or 1 where 0 represents the white color and 1 to the black one.

\section{Input Parameter:}

$\begin{array}{lll}\text { EPOCH : } & 10 & \\ \text { Learning Rate } & : & 0.05 \\ \text { LR Reduction } & : & 0.1\end{array}$

\section{Training Result:}

$\mathrm{EPOCH}: 1$

$\begin{array}{lll}\text { ========== }========== \\ \text { W1 } & : & 96,2029105588807 \\ \text { W2 } & : & 63,6945837571767 \\ \text { W3 } & : & 71,6449579523919 \\ \text { Max } & : & \text { W3 } \\ & & \\ \text { W1 } & : & 63,6945837571767 \\ \text { W2 } & : & 85,463442476886 \\ \text { W3 } & : & 63,3506116781836 \\ \text { Max } & : & \text { W3 } \\ \text { W1 } & : & 71,6449579523919 \\ \text { W2 } & : & 63,3324561342761 \\ \text { W3 } & : & 92,7327207624153 \\ \text { Max } & : & \text { W2 }\end{array}$

EPOCH : 2

\begin{tabular}{|c|c|c|}
\hline W1 & : & 96,2029105588807 \\
\hline W2 & : & 64,1155207418617 \\
\hline W3 & : & 72,6291608102379 \\
\hline Max & : & W3 \\
\hline W1 & : & 63,6945837571767 \\
\hline W2 & : & 84,4946743883874 \\
\hline W3 & : & 64,5940974470564 \\
\hline Max & : & W1 \\
\hline W1 & : & 71,2917246249516 \\
\hline W2 & : & 65,2859096589798 \\
\hline W3 & : & 91,8878197178523 \\
\hline Max & : & W2 \\
\hline
\end{tabular}

EPOCH : 3

\begin{tabular}{|c|c|c|}
\hline W1 & : & 94,979418823236 \\
\hline W2 & : & 64,4732425119125 \\
\hline W3 & : & 73,8518464562668 \\
\hline Max & : & W3 \\
\hline W1 & : & 64,8314352764163 \\
\hline W2 & : & 83,6574817335572 \\
\hline W3 & : & 64,5579106638941 \\
\hline Max & : & W3 \\
\hline W1 & : & 71,2917246249516 \\
\hline W2 & : & 66,9109015781391 \\
\hline W3 & : & 90,1954553444742 \\
\hline Max & : & $\mathrm{W} 2$ \\
\hline
\end{tabular}


EPOCH : 4

\begin{tabular}{|c|c|c|}
\hline W1 & : & 94,979418823236 \\
\hline W2 & : & 64,7791258083964 \\
\hline W3 & : & 74,4662009083135 \\
\hline $\operatorname{Max}$ & : & W3 \\
\hline W1 & : & 64,8314352764163 \\
\hline W2 & : & 82,9311611692216 \\
\hline W3 & : & 65,4681394369323 \\
\hline $\operatorname{Max}$ & : & W1 \\
\hline W1 & $:$ & 71,0172757890635 \\
\hline W2 & : & 68,2766831767992 \\
\hline W3 & : & 89,586769334485 \\
\hline Max & : & $\mathrm{W} 2$ \\
\hline
\end{tabular}

EPOCH : 5

\begin{tabular}{|c|c|c|}
\hline W1 & : & 94,0220661307755 \\
\hline W2 & : & 65,0421138542461 \\
\hline W3 & : & 75,3686721266498 \\
\hline Max & : & W3 \\
\hline W1 & : & 65,6973535102458 \\
\hline W2 & : & 82,2987894398208 \\
\hline W3 & : & 65,4107208423767 \\
\hline Max & : & W3 \\
\hline W1 & : & 71,0172757890635 \\
\hline W2 & : & 69,4344907189694 \\
\hline W3 & : & 88,330624672591 \\
\hline Max & : & $\mathrm{W} 2$ \\
\hline
\end{tabular}

\begin{tabular}{|c|c|c|}
\hline W1 & : & 94,0220661307755 \\
\hline W2 & : & 65,269317872934 \\
\hline W3 & : & 75,7667249725151 \\
\hline Max & : & W3 \\
\hline W1 & : & 65,6973535102458 \\
\hline W2 & : & 81,7464394113101 \\
\hline W3 & : & 66,0934192726768 \\
\hline Max & : & $\mathrm{W} 1$ \\
\hline W1 & : & 70,8023360370648 \\
\hline W2 & : & 70,4231262073374 \\
\hline W3 & : & 87,8833859679634 \\
\hline Max & : & W2 \\
\hline
\end{tabular}

EPOCH : 7

$\begin{array}{lll}\text { =========== }============ \\ \text { W1 } & : & 93,268048329676 \\ \text { W2 } & : & 65,4664514717543 \\ \text { W3 } & : & 76,4483983864839 \\ \text { Max } & : & \text { W3 } \\ \text { W1 } & : & 66,3653317941401 \\ \text { W2 } & : & 81,2625758553348\end{array}$




$\begin{array}{lll}\text { W3 } & : & 66,0308043811068 \\ \text { Max } & : & \text { W3 } \\ & & \\ \text { W1 } & : & 70,8023360370648 \\ \text { W2 } & : & 71,2725458245539 \\ \text { W3 } & : & 86,9358127091439 \\ \text { Max } & : & \text { W1 }\end{array}$

EPOCH : 8

$\begin{array}{lll}\text { ======= }============== \\ \text { W1 } & : & 92,7586912359887 \\ \text { W2 } & : & 65,4664514717543 \\ \text { W3 } & : & 76,7142975886221 \\ \text { Max } & : & \text { W3 } \\ & & \\ \text { W1 } & : & 66,2865367571388 \\ \text { W2 } & : & 81,2625758553348 \\ \text { W3 } & : & 66,5520464354352 \\ \text { Max } & : & \text { W1 } \\ \text { W1 } & : & 71,3677269180747 \\ \text { W2 } & : & 71,2725458245539 \\ \text { W3 } & : & 86,6016510553595 \\ \text { Max } & : & \text { W2 }\end{array}$

EPOCH : 9

\begin{tabular}{|c|c|c|}
\hline W1 & : & 92,1706559556189 \\
\hline W2 & : & 65,6209995301784 \\
\hline W3 & : & 77,2377788803101 \\
\hline Max & : & W3 \\
\hline W1 & : & 66,8094261851265 \\
\hline W2 & : & 80,8801858207332 \\
\hline W3 & : & 66,4918373225648 \\
\hline Max & : & W3 \\
\hline W1 & : & 71,3677269180747 \\
\hline W2 & : & 71,9332209561111 \\
\hline W3 & : & 85,877285998367 \\
\hline Max & : & $\mathrm{W} 1$ \\
\hline
\end{tabular}

EPOCH : 10

\begin{tabular}{|c|c|c|}
\hline W1 & : & 91,7772250077996 \\
\hline W2 & : & 65,6209995301784 \\
\hline W3 & : & 77,4200680670207 \\
\hline Max & : & W3 \\
\hline W1 & : & 66,7364976522469 \\
\hline W2 & : & 80,8801858207332 \\
\hline W3 & : & 66,8951161322839 \\
\hline Max & : & W1 \\
\hline W1 & : & 71,8025165865998 \\
\hline W2 & : & 71,9332209561111 \\
\hline W3 & : & 85,6240631534816 \\
\hline Max & : & $\mathrm{W} 1$ \\
\hline
\end{tabular}

There are ten loops for EPOCH $=10$ and each loop consists of three classes. Every clase has a maximum weight. After the training result has been done, the test for the fingerprint is performed. 


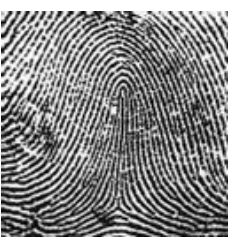

Fig. 9 Test fingerprint 1

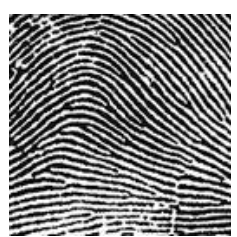

Fig. 10 Test fingerprint 2

Figure 9 and 10 are the test fingerprints. They will be tested to the training data where it would be closer.

Test Result:

TEST : 1 (Figure 9)

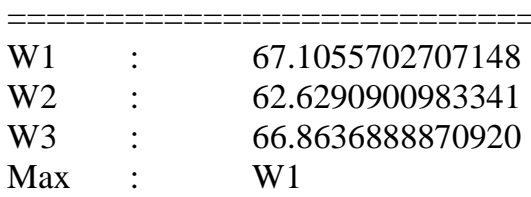

TEST : 2 (Figure 10)

\begin{tabular}{|c|c|c|}
\hline W1 & : & 70.2808924695356 \\
\hline W2 & : & 64.5118337314814 \\
\hline W3 & $:$ & 69.3663784430469 \\
\hline Max & : & W1 \\
\hline
\end{tabular}

From both tests, the result of each test is close to the Arch fingerprint. If it is seen by naked eyes, it is close to the Arch as well (Figure 2 and Figure 6). It proved that the test is highly accurate.

\section{Conclusion}

The development of a classification system fingerprint patterns for identification via fingerprint acquisition stages and phases of classification has a significant role to assist in the investigation related to the digital data. Acquisition stages using a fingerprint scanner, followed by fingerprint feature extraction, the training process, the process of classification and identification of personality based on the pattern classification results. LVQ algorithm has a good degree of fit to recognize the fingerprint pattern is more inclined to go. This level of accuracy can be set to specify the input parameters include EPOCH, Learning Rate, and LR Reduction. By determining the combination of a good result was obtained closer to the truth.

\section{References}

[1]. P. Hájek, "Fuzzy Logic and Arithmetical Hierarchy," Fuzzy Sets and Systems, vol. 73, pp. 359-363, 1994

[2]. A. P. U. Siahaan, "Fuzzification of College Adviser Proficiency Based on Specific Knowledge," International Journal of Advanced Research in Computer Science and Software Engineering, vol. 6, no. 7, pp. 164-168, 2016.

[3]. S. B. N., B. M. Bannur, B. G. Patil, G. A. Hadimani and D. P. Jose, "Dermatoglyphic Patterns in Patients of Bronchial Asthma: A Quantitative Study," International Journal of Biological \& Medical Research, vol. 2, no. 1, pp. 38-42, 2013.

[4]. S. Prasad, V. K. Singh and Akshay, "Handwriting Analysis based on Segmentation Method for Prediction of Human Personality using Support Vector Machine," International Journal of Computer Applications, vol. 8, no. 12, pp. 25-29, 2010.

[5]. S. Yohannes, T. Hundessa, B. Duale, B. Getahun, H. Ali and D. Negasa, "Dermatoglyphic Biomarkers and Functional Handedness in Multiple Intelligence: A University Based Cross-Sectional Study," International Journal of Current Research, vol. 7, no. 8, pp. 19278-19283, 2015.

[6]. M. H. Bhuyan and D. K. Bhattacharyya, "An Effective Fingerprint Classification and Search Method," International Journal of Computer Science and Network Security, vol. 9, no. 11, pp. 39-68, 2009.

[7]. B. C. Arabacioglu, "Using Fuzzy Inference System For Architectural Space Analysis," Applied Soft Computing, vol. 10, p. 926937, 2010.

[8]. L. Biacino and G. Gerla, "Fuzzy Logic, Continuity and Effectiveness," Mathematical Logic, vol. 41, p. 643-667, 2002.

[9]. G. Gerla, "Effectiveness and Multivalued Logics," The Journal of Symbolic Logic, vol. 71, pp. 137-162, 2006.

[10]. S. S. Jamsandekar and R. R. Mudholkar, "Fuzzy Classification System by Self Generated Membership Function Using Clustering," International Journal of Information Technology, vol. 6, no. 1, pp. 697-704, 2014. 